\title{
Quantum Chemistry of in situ Retinal: Study of the Spectral Properties and Dark Adaptation of Bacteriorhodopsin ${ }^{\dagger}$
}

\author{
Ilya Logunov* and Klaus Schulten \\ Beckman Institute and Departments of Chemistry and Physics, \\ University of Illinois at Urbana-Champaign, \\ 405 North Matthews, Urbana, Illinois 61801
}

October 12, 1995

$\dagger$ This work was supported by grants from the National Institutes of Health (PHS 5 P41 RR0596904), the National Science Foundation (BIR-9318159), and the Roy J. Carver Charitable Trust.

* To whom correspondence should be addressed. Email: ilya@ks.uiuc.edu 
A combination of molecular dynamics and quantum chemistry techniques have been employed to study the electronic excitation and conformational potential surface of retinal in the binding site of bacteriorhodopsin (bR). The CASSCF $(6,9) / 6-31 \mathrm{G}$ level of ab initio calculations (within Gaussian92) has been used for the treatment of both the ground (S0) and excited (S1) states of retinal. Charges of all atoms in the protein are represented by spherical Gaussians and explicitly included in the electronic Hamiltonian of retinal. Spectral properties have been analyzed for the native bR pigment as well as for its D85N mutant. The calculated relative shift in the absorption maxima between the two pigments is in better agreement with experiment than the computed absolute parameters of the absorption line shapes. The dark adaptation processes in bacteriorhodopsin (which involves rotation around the 13-14 and the 15-N retinal double bonds) has been modelled by following the pre-defined reaction coordinate. Our simulations support the notion that the isomerization process is catalyzed by the protonation of an aspartic acid (Asp85) side group of bacteriorhodopsin. 


\section{Introduction}

Bacteriorhodopsin (bR) spans the cell membrane of Halobacterium halobium and functions as a light-driven proton pump. bR contains seven $\alpha$-helices which enclose the prosthetic group, all-trans retinal, bound via a protonated Schiff base linkage to Lys-216. Figure 1a shows the chemical structure of retinal and its conventional numbering scheme. The bR structure is presented in Fig. 2a. Retinal absorbs light and undergoes a photoisomerization process; the thermal reversal of this reaction is coupled to transfer of a proton from the cytoplasmic side (top in Fig. 2a) to the extracellular side (bottom in Fig. 2a) of the protein. Recent reviews that discuss the structure and function of bR are $[1,2,3,4,5,6,7]$.

Bacteriorhodopsin accomplishes its function through a cyclic process initiated by absorp- here tion of a photon. This photon triggers an isomerization of retinal, which proceeds then through several intermediate states identified by their absorption spectra. An accepted kinetic scheme for this cycle is an unbranched series of intermediates, shown in Fig. 3. Photoisomerization occurs in the $\mathrm{bR}_{568} \rightarrow \mathrm{J}_{625}$ transition. During the $\mathrm{L}_{550} \rightarrow \mathrm{M}_{412}$ transition the Schiff base proton is transferred to Asp- 85 and, subsequently, to the outside of the cell $[8,9,10,11,12,13]$. During the $\mathrm{M}_{412} \rightarrow \mathrm{N}_{520}$ transition, a proton is transferred to the Schiff base from Asp-96, which then takes up a proton from the cytoplasmic environment [13]. The reaction cycle is completed as the protein returns to $\mathrm{bR}_{568}$ via the $\mathrm{O}_{640}$ intermediate.

When bR is allowed to equilibrate in the dark, it converts within an hour to a 2:1 here mixture containing 13-cis retinal and all-trans retinal bR[14]. The protein containing the 13-cis retinal isomer of bRis referred to as the dark adapted (DA) pigment of bR, bR $R_{548}$, which absorbs at $548 \mathrm{~nm}$. bR $\mathrm{b}_{548}$ contains, actually, retinal in a 13-cis,15-syn geometry as 
suggested first in $[15]$ and observed in $[16,17,18] . \mathrm{bR}_{548}$ undergoes a light-driven photocycle similar to that of all-trans retinal which also involves an initial photoisomerization about the $\mathrm{C}_{13}-\mathrm{C}_{14}$ double bond (see Fig. 3). However, the respective photocycle does not result in vectorial proton translocation. The detailed description of this, so-called cis-photocycle can be found in $[19,20,21,22,23,24]$.

A widely accepted model for the three-dimensional structure of bR has been provided here by Henderson and coworkers using electron-microscopy at low temperature [25, 26] together with bR's amino acid sequence [27, 28]. The obversations resulted in a structure for the membrane-spanning helical portion of bR at a resolution of $3 \AA$ in a direction parallel to the membrane and at a resolution of $10 \AA$ perpendicular to the membrane. This structure provided an opportunity to explore, by means of computer simulations, the mechanism of bR's light-driven proton pump at the atomic level. Molecular dynamics techniques have been widely used to refine the Henderson structure and to study structural properties of the $\mathrm{bR}_{568}$ pigment and its photocycle intermediates [29, 30, 31, 32, 33, 34, 35].

While molecular dynamics is a very powerful tool for structural refinement, the realm of questions which can be addressed by this technique is rather limited. Processes like photoand thermally- induced isomerization of in situ retinal, as well as the proton transfer processes in bR (see Fig. 3), require detailed potential surfaces which need to be provided by quantum chemical methods. Quantitative prediction of the experimentally observed spectra of bR and its photocycle intermediates (see Fig. 3) also necessitate the use of quantum chemical calculations. However, such calculations need to be combined with classical molecular dynamics simulations to account for the effect of a thermal environment. 


\section{Quantum Chemistry of in situ Retinal}

The description of the spectra and extremely rapid photodynamics of visual pigments and bacteriorhodopsin has been a major endeavor for quantum chemists. Until recently, only crude models could be applied in the study of the potential surfaces $[36,37,38,3]$ and spectral properties $[39,40,41,42,43,44]$ of in situ retinal, due to the lack of the necessary atomic structures. The determination of a low resolution structure of bR and its refinement by means of molecular dynamics simulations allows one now to explore the electronic properties and chemical transformations of retinal when bound within the apoprotein.

Major challenges associated with the ab initio electronic structure calculations of in situ retinal still remain. A first complication originates from the strongly correlated nature of the conjugated $\pi$-electron system of retinal. Retinal belongs to the family of polyene dyes, which are well known for their characteristic structure of alternating single and double $\pi$ electron bonds. Several research groups have studied the electronic structure and potential surfaces of conjugated polyene systems [39, 45, 46, 47, 48, 49, 50, 51]. A distinct feature of the electronic structure of a conjugated polyene is the existence of near-degenerate excited states, and crossing between different potential surfaces for the twisted geometries of a carbon chain $[52,53,51,54,55]$. The most efficient way of allowing for the existence of near degenerate states in quantum chemical calculations is through implemention of the multiconfigurational (MCSCF) treatment $[56,57,58,59]$. Correlation effects can be taken into account more completely by performing configuration interaction (CI) calculations [60, 61,

$62,63,64,65]$. However, the use of CI calculations on top of an ab initio MCSCF treatment is computationally prohibitive for almost any length of a carbon chain. An alternative and 
very promising approach is implemetation of multi-reference pertubation methods, such as CASPT2 [66, 67, 68].

Another difficulty arises from the complexity of taking into account the protein environment when studying the electronic structure of retinal in bR. It has been observed that modification of protein groups in the vicinity of the retinal Schiff base shifts considerably the bR absorption spectrum [69] and affects drastically the rates of both thermally- [70] and photo- $[71,72]$ activated isomerization processes in bR. The experiments imply that the protein environment plays a crucial role in determining the physico-chemical properties of retinal in situ.

The problem of the representation of the environment in electronic structure calculations of a molecule in situ is very complex. Initially, a combination of in vacuo ab initio quantum chemical and classical solvation energy calculations $[73,74]$ was implemented. Later, various techniques were developed which allow for the self-consistent ab initio treatment of a molecule embedded in a dielectric continuum $[75,76,77]$. At present, there exist a number of ab initio as well as semi-empirical techniques which try to account for the realistic atomic structure and charge distribution of the environment via explicit incorporation of protein (or solution) point charges in the electronic Hamiltonian of the quantum chemically treated substrate $[78,79,80,81,82,83]$

In our study, the refined structure of bacteriorhodopsin reported in [31] was used as a starting point for all the computations. This structure, corresponding to the $\mathrm{bR}_{568}$ pigment, is depicted in Figures 2a and 5b. Figure 2a provides a view of the protein backbone, water, and retinal. The Schiff base region of the retinal binding site is shown in Figure 5b. The 
figure shows the relative position of the major active groups in the binding site. The key protein groups which affect the electronic structure of retinal are the two negatively charged aspartic acids, Asp-85 and Asp-212, as well as the positively charged Arg-82. Water molecules bound within the bR active site are also of importance, particularly a water which is directly hydrogen bound to the Schiff base $\mathrm{NH}^{+}$group and plays the role of the primary counter-ion (see Fig. 5b).

All the groups within the retinal binding site as well as retinal itself are in a constant thermal motion. The dynamics plays a major role in regulating the physico-chemical properties of in situ retinal along with the structural and stereochemical factors. Shown in Figures 2 are time-dependent properties of in situ retinal. The examples are given for the internal degrees of freedom of retinal (1b) and its interaction with the environment (2b). The torsional degrees of freedom of retinal undergo significant thermal fluctuations. The torsions about individual bonds can vary within a range of a few tens of degrees, affecting significantly the energetics of the $\mathrm{S} 0 \rightarrow \mathrm{S} 1$ transition and the retinal isomerization potential surface of retinal. The variation of the total retinal dihedral energy with time is also shown in Figure 2. It is seen that the amplitude of the dihedral energy fluctuations is roughly $6 \mathrm{kcal} / \mathrm{mole}$, i.e., approximately thirty percent of the average value $(18 \mathrm{kcal} / \mathrm{mole})$. Shown in Figure 2 is furthermore the time dependence of the electrostatic interaction energy between retinal and the rest of the protein. Absolute fluctuations in this case are significantly larger than those for the retinal internal energy and constitute about $25 \mathrm{kcal} / \mathrm{mole}$. These numbers indicate that rigorous treatment of the dynamics of the protein interior and proper accounting for a statistical ensemble of protein structures are of primary importance. 
To this end, we have begun a major effort to compute the electronic structure of in situ retinal using standard, ab initio correlated electronic structure techniques combined with a classical description of the protein environment. These calculations are the first of their kind for bR. So far, ab initio techniques have been employed only for the calculation of charge distribution and geometric parameters of in situ retinal [84]. Described below are two examples of combined quantum/classical studies of the ground state potential surface and spectral properties of retinal in the bR binding site. 


\section{Modelling Spectral Properties of Bacteriorhodopsin}

While, at first, retinal seems rather large for modern, correlated electronic structure techniques, in fact, because the chromophore is protonated while residing inside the protein, computations of the excited state absorption spectra require only a single CASSCF $a b$ initio calculation to obtain both the ground and excited state energies. In contrast, polyenes require for the description of their spectra more extended calculations due to a near degeneracy of an ionic, optically allowed excited state, a covalent, optically forbidden excited state and admixtures of diffuse atomic orbitals. As a result, the excited states of long chain polyenes are typically hard to locate in electronic structure calculations. However, for the protonated Schiff base of retinal, the excited state resembles the ground state much more than a typical polyene [40], and the lowest, optically allowed singlet state of retinal is easily detected in in vacuo electronic structure calculations. (For a more detailed discussion, see [47].) Hence, single excited state CASSCF calculations (specifically, CASSCF(6,9)/6-31G $a b$ initio calculations using Gaussian92, optimized for the first singlet excited state) describe well the optically allowed excitation energy. The excitation energy is thus the difference between the second and the first roots of the CI matrix.

Ab initio calculations have been performed on a modified retinal analog shown in Fig. 1(b) with the $\beta$-ionone ring and two methyl groups removed. Identical calculations have been performed on a smaller $\mathrm{CH}_{2} \mathrm{CHCHNH}_{2}$ analog, and the vertical excitation energies agree to within $0.2 \mathrm{eV}$ with both multi-reference single- and double-configuration calculations (MRSDCI) $[85,47,55]$ and with effective valence shell Hamitonian calculations. The charges of all atoms in the protein were represented by spherical Gaussians (with a half-width of two 
$\AA)$ and were explicitly included in the electronic Hamiltonian of retinal.

Many different factors affect the absorption spectrum of in situ retinal. At any moment in time, the transition energy $\Delta E(t)$ between the ground and the excited states assumes a value determined by the momentary orientations of protein side groups as well as depends on the conformation and vibrational excitation of retinal. This leads to both homogeneous and inhomogeneous broadening of $\Delta E(t)$. Molecular dynamics simulations combined with ab initio quantum chemistry calculations allow one to determine both equilibrium properties, e.g., the average value and equilibrium distribution of $\Delta E(t)$ values, as well as dynamical properties, e.g., the autocorrelation function of $\Delta E(t)$.

Figure 4 presents the time dependence of the energy of the $\mathrm{S}_{o} \rightarrow \mathrm{S}_{1}$ transition in retinal in bR during a 100 ps dynamical trajectory. The absorption spectral line shape is computed by performing quantum chemical calculations accounting for charge distributions obtained from MD trajectories, taking snapshots every picosecond. The resulting distribution of $\Delta E(t)$ values have been matched to a Gaussian, ignoring statistically insignificant data, the Gaussian distribution being parameterized by the average value and by the RMSD of $\Delta E(t)$. Figure 4

Figure 5a compares calculated and observed [2] absorption line shapes. Fitting the tran- here sition energies to a Gaussian distribution yields a curve with a maximum at $524 \mathrm{~nm}$ and a RMSD of $43 \mathrm{~nm}$. This result compares well with the experimentally observed absorption maximum at $568 \mathrm{~nm}$ and a RMSD of $34 \mathrm{~nm}$. We have carried out an equivalent calculation for the mutant D85N. The retinal binding site structure of this mutant is shown in Fig. 5. The computed absorption maximum of the D85N mutant, also shown in Fig. 5, lies $50 \mathrm{~nm}$ to the red of the absorption maximum of native bR, the shift agreeing closely with the 
experimentally observed $47 \mathrm{~nm}$ red shift [69]. This agreement is quite remarkable considering the number of approximations made in treating the protein environment. However, the errors introduced seem to cancel systematically, expecially when computing the absorption spectral shift. This is a common feature of ab initio calculations, as energy differences (and differences of energy differences) are easier to compute than absolute energies.

The structures of bR and the D85N mutant are compared in Fig. 5(b). Although the replacement of Asp-85 by Asn noticably changes the overall structure of the retinal binding pocket, the red shift in the absorption maximum of D85N mutants most likely results from the lower negative charge of the Schiff base counterion complex. Such an explanation is in accordance with the general concept that the proximity of a negative charge to the protonated Schiff base leads to a blue shifted excitation [39]. The effect is rationalized as being due to the transfer of negative electronic density to the Schiff base region in the excited state, thus making Coulomb interactions with a negatively charged counterion less favorable as compared to the ground state interactions of retinal. This rationalization is supported by the results of a Mulliken populational analysis of the electronic densities in both the ground and excited states which shows that upon excitation about 0.4 of an electron is tranferred to the Schiff base moiety of retinal.

An important factor which has been left out in our calculations is the electronic po- here larizability of the retinal protein environment. Transfer of 0.4 of an electron to the Schiff base region upon exciation implies that photo-excitation of retinal leads to an alteration of the electric field in its vicinity and, thus, to an additional polarization of the neighbouring protein groups. This electronic polarization is instantaneous, i.e. it occurs on the same time 
scale as an elementary act of the photon absorption, thus directly affecting the transition energy. An instantaneous electronic polarization of the environment leads to a further red shift in the absorption due to additional stabilization of the electronic excited state. The neglect of the protein polarizabilities can be partially responsible for the blue shift of the computed absorption line shapes as compared with the experimentally observed ones. This neglect may also be responsible for the overestimation of the spectral width of retinal in our calculations (see below).

A number of dynamical factors affect the electronic absorption spectrum of retinal in situ, including the intereaction between the Schiff base and counterion residues, the electrostatic interactions with the protein interior, and the internal geometry of retinal itself. These factors reveal themselves in an analysis of the correlation function of the energy of the $\mathrm{S}_{0} \rightarrow \mathrm{S}_{1}$ electronic transition. Figure 4 examines the correlation between the energy of the electronic transition and the strength of hydrogen-bonding between water molecules and the Schiff base $\mathrm{N}-\mathrm{H}^{+}$group. In our model structure, a water molecule serves as a primary counterion to the positively charged protonated Schiff base. Correspondingly, decreases in the excitation energy strongly correlate with an increased distance between the water oxygen and the Schiff base nitrogen (on a 100 ps time scale), as demonstrated by both the time evolution of the N-O distance (see Fig. 4(1a,b)) as well as in the overall shape of the corresponding auto-correlation functions (see Fig. 4(2a,b)).

Both fluctuations of the retinal environment and the torsional motions of retinal lead to the boadenning of the absorption spectrum. The calculated width of the bR absorption spectrum compares well with the experimentally observed one, exceeding it by about twenty 
percent (see Fig. 5). It should be emphasized, though, that only 'inhomogeneous' broadening, i.e., broadening due to possible different conformations of the chromophore and its environment has been taken into account in our simulations. However, the experimentally observed line shape incorporates two different types of broadening. Along with 'inhomogeneous' broadening, there exists 'homogeneous' brodenning which is due to the transitions between different vibrational levels of the ground and excited electronic states in retinal. The 'vibrational structure' of the bR spectrum consists of three major and a few minor [86] 'vibrational bands'. In case of the $\mathrm{bR}_{568}$ spectrum, the 'vibrational structure' is manifested in the asymetrical nature of the experimentally observed absorption line shape (Fig. 5). The 'vibrational broadenning' is an essentially quantum effect, which can be properly taken into account by performing vibrational normal mode calculations for the ground and excited electronic states of retinal and computing the Frank-Condon coefficients. Calculations presented here also incorporate, to some extent, the vibratinal motions of retinal due to classical thermal fluctuations. Although these internal vibrations of retinal lead to an additional broadennig of the absorption line shape, their effect can be quite different from the one given by the Frank-Condon coefficients. 


\section{Modeling the Dark Adaptation of Bacteriorhodopsin}

Many important functions of bR proceed through the thermally activated isomerization of retinal around one or two of its double bonds; such functions include the conversion of 13-cis retinal to the all-trans configuration during the later stages of the pump cycle, the process of dark adaptation in bR, and a possible leakage pathway from the 13-cis (dark adapted) to the all-trans (light adapted) photocycle. While standard MD force fields can describe the ground state isomerization only for assumed models of the isomerization barriers, current computational facilities permit a partial quantum mechanical treatment of the ground state potential surface of in situ retinal, avoiding an a priori choice of an isomerization barrier. A full $a b$ initio description still remains out of reach, and we can at best hope to model the isomerization process of in situ retinal by treating the chromophore electronic degrees of freedom quantum mechanically and the protein environment along with its dynamics classically. The current section describes our efforts to develop a mixed ab initio quantum mechanical/classical simulation. We apply our simulations to study the dark adaptation process in bR, which involves a reversible thermally activated transformation of retinal from an all-trans to a 13-cis,15-syn configuration as shown in Fig. 6.

The ground state potential surface of retinal has been computed within Gaussian92 using here the CASSCF $(6,9) / 6-31 \mathrm{G}$ level of ab initio calculations. Calculations have been performed on a modified retinal analog (Fig. 1(b)) with the $\beta$-ionone ring and two methyl groups removed. Charges of all atoms in the protein are represented by spherical Gaussians (with a half-width

of two $\AA$ ) and are explicitly included in the electronic Hamiltonian of retinal. The in situ isomerization is modelled by following a pre-defined reaction coordinate (co-rotation around 
the $\mathrm{C}_{13}-\mathrm{C}_{14}$ and $\mathrm{C}_{15}-\mathrm{N}$ retinal double bonds combined with a complete minimization of the whole protein). In calculating the in vacuo potential surface of retinal, the same sequence of retinal conformations have been chosen without taking into account the protein charge environment.

An example of the retinal dark adaptation potential surface for a particular snapshot structure is shown in Fig. 7a. It is seen that the placement of retinal inside the protein reduces the isomerization barrier governing the thermal isomerization of retinal around two $\left(\mathrm{C}_{13}-\mathrm{C}_{14}\right.$, $\mathrm{C}_{15}-\mathrm{N}$ ) double bonds, by about $20 \mathrm{kcal} / \mathrm{mol}$. This result is in agreement with the common notion that the protein environment enhances the isomerizational transformations of in situ retinal $[71,72]$. However, the question arises whether the standard classical electrostatic models with their pairwise additive potentials describe the chromophore-protein interactions properly, or if the polarizing effect of the environment must be included. Figure 7b shows the effect of Coloumb interactions in the retinal binding site on the isomerization potential surface computed by a combination of classical MD and quantumchemical calculations.

The classical Coulomb energy is the sum of all electrostatic interactions between the here retinal molecule and the rest of the protein, using the standard MD point charge model. The quantum 'interaction' energy is the difference between in situ and in vacuo potential energy profiles given in Fig. 7b and, therefore, includes the polarizing effects of the protein environment. The classical MD pairwise-interactions, in fact, slightly inhibit the isomerization process, in direct contrast to the results of ab initio calculations. It can be concluded that the standard MD force field, which neglects changes of retinal's electronic structure, fails to represent the potential surface of in situ retinal. In contrast, the ab initio calcula- 
tions clearly demonstrate that the protein pocket does lower the retinal isomerization energy barrier. This effect can be rationalized as being due to the repulsion of the electron cloud from the Schiff base region by the negative counterion complex of the bR binding site, which results in the decrease of the double bond character of the $\mathrm{C}_{13}-\mathrm{C}_{14}$ and $\mathrm{C}_{15}-\mathrm{N}$ bonds. Such an explanation is supported by the results of a Mulliken populational analysis which shows that upon placement of retinal in the bR binding pocket about 0.2 units of electron density is withdrawn from the Schiff base region of retinal.

It has been found that the potential barrier for dark adaptation depends on the momentary protein structure. In order to evaluate the overall rate of the dark adaptation proccess one must consider an ensemble of trans-bR structures and determine the distribution of energy barriers. Shown below is one possible way of tackling the problem of multiple energy barriers.

Effective Energy Barrier of Dark Adaptation In order to determine the kinetics of the dark adaptation process, the isomerization potential barriers were computed for a number of different structures selected at equal time intervals from an MD trajectory. The obtained distribution of barrier heights was approximated by a Gaussian, parameterized by the average value $E_{0}$ and the RMSD $\sigma$ of the computed barrier heights. By integrating over an assumed Gaussian distribution of barriers, we obtain the effective activation energy, which is related to the overall rate of the dark adaptation process. For this purpose we obtain the expression for the rate of dark adaptation 


$$
k=k_{0} \int p(E) \exp \left(-\frac{E}{k T}\right) d E ; \quad \int p(E) d E=1
$$

Here $p(E)$ is the distribution of barrier hights.

As stated above, $p(E)$ is modelled by a Gaussian distribution; however, in order to obtain a consistent result for any possible Gaussian distribution, one has to assume in the integrals (1) that an activation energy cannot be smaller than some minimal value $E_{\min } . E_{\min }$ is taken as a minimal activation barrier among all considered structural snapshots. Our simulations yield $E_{\min }=21 \mathrm{kcal} /$ mole at $T=300 \mathrm{~K}$. Accordingly, we expess the rate constant of dark adaptation

$$
k=k_{0} \frac{1}{\sqrt{2 \pi} \sigma} \int_{E_{\min }}^{\infty} \exp \left[-\frac{1}{2 \sigma^{2}}\left(E-E_{0}\right)^{2}\right] \exp \left[-\frac{E}{k T}\right] d E
$$

This expression is matched to the conventional Arrenius expression

$$
k=k_{0} \exp \left[-\frac{E_{\mathrm{eff}}}{k T}\right]
$$

where $E_{\text {eff }}$ can be temperature dependent. There exist two limiting cases. In case of $E_{0}>>\sigma^{2} / R T$ one approximates

$$
E_{\text {eff }} \approx E_{0}-\sigma^{2} / 2 R T
$$

In case of $E_{0}<<\sigma^{2} / R T$ one approximates

$$
E_{\text {eff }} \approx E_{\min }
$$

Many experiments suggest that the dark adaptation process in bR is catalyzed by protonation of the Asp-85 side group [70] (see Fig. 6). To test this hypothesis we have carried 
out simulations for both deprotonated and protonated states of Asp-85. The resulting distributions of the energy barriers for both protonation states of Asp- 85 are shown in Figure 8. When Asp-85 is deprotonated, we obtain $E_{0}=39 \mathrm{kcal} / \mathrm{mole}, \sigma=4 \mathrm{kcal} / \mathrm{mole}$, yielding according to $(4)$

$$
E_{\text {eff }}=26 \mathrm{kcal} / \mathrm{mole} \text {. }
$$

When Asp-85 is protonated, we obtain $E_{0}=31 \mathrm{kcal} / \mathrm{mole}, \sigma=8 \mathrm{kcal} / \mathrm{mole}$, yielding according to (5)

$$
E_{\text {eff }}=21 \mathrm{kcal} / \mathrm{mole} \text {. }
$$

It follows that protonation of Asp-85 increases the dark adaptation reaction rate by a few orders of magnitude, which is in good agreement with experiment.

In general, our study supports the notion that Coulomb interactions play a major role here in governing chemical transformations in a protein $[82,87,88,89,90]$. However, Coulomb interactions cannot be properly accounted for by mere pairwise potentials of classical electrostatics, since the protein charge environment polarizes significantly the substrate and alters its electronic structure. It can also be concluded that protein thermal motions significantly broaden the energy distribution which enters into the rate equations for in situ chemical transformations. 


\section{Further Developments}

One of the major challenges in the field of $\mathrm{bR}$ modelling is the quantitative prediction of the experimentally observed spectral properties and photo-reactivity of bR. These require a combination of quantum chemical and molecular dynamics calculations. Many efforts are being invested at present into merging molecular dynamics and quantum chemistry techniques $[81,83,91,87,90,84,92]$. The importance of combining these two major tools of computational chemistry is now being realized to a large extent by both the molecular dynamics and the quantum chemistry communities.

In our studies we have applied a combination of quantum chemistry and molecular dynamics techniques to study the spectral properties and isomerization potential of in situ retinal. Our first attempt to use a combined quantum/classical mechanical approach to study electronic properties of bR can be considered successful. We have been able to describe satisfactorily the spectral properties of bR as well as the thermally activated isomerization of in situ retinal responsible for the dark adaptation of bR. This accomplishment paves the road towards further simulation of the entire bR photocycle by means of combined quantum/classical techniques.

The most fascinating process associated with the bR proton pump mechanism is the primary photoisomerization reaction (see Fig. 3). It occurs on a time scale of a few hundred femtoseconds and constitutes the fastest chemical process known in biology. The primary

photochemical event is typical not only for bR, but is shared by all proteins incorporating the retinal prosthetic group. In situ photoisomerization of retinal is quite different from that in solution in the sense that the arrangement of the protein side groups determines both the 
stereochemistry and the time scale of the primary photochemical event.

Another chemical process which is essential for the function of $b R$ is the proton transfer reaction. The overall proton pump activity of bR results from a series of elementary proton transfer processes performed mainly in the same vectorial direction from inside to outside of the cell (see Fig. 3). It is important to understand on a quantitative level the major driving forces which determine this vectorial transfer of a proton across the membrane and to be able to predict the rates of the individual proton tranfer steps which can be measured experimentally. The problem of adequate modelling of in situ proton transfer reactions extends far beyond the bR proton pump cycle itself, since it is one of the most common elementary chemical process to be found in a living cell (see, e.g., [93, 94]) .

It is evident from the present study that further improvements in both the level of quantum chemical $a b$ initio calculations and the accuracy of bR models are needed in order to describe quantitatively the wide range of experimentally observed bR properties. As was pointed out ealier, both static (due to state degeneracies) and dynamic (due to electronelectron pairwise interaction) correlation effects are of importance when considering the electronic structure of conjugated polyene molecules such as retinal. However, the CASSCF level of $a b$ initio calculations employed in the present study accounts only for the static correlation effects due to the near degeneracy of a few close-lying electronic states. Dynamic correlation has not been accounted for properly in the present study. To account for the correlated elecron-electron interactions, multi-reference CI or multi-reference perturbation methods need to be implemented on top of MCSCF calculations.

The question of an adequate representation of the protein environment in performing in 
situ ab intio calculations has not yet been resolved completely. The diffuse charge model employed in the present calculations can be considered as an improvement of the previously used point charge models. Indeed, in the particular case of in situ ab initio calculations of the retinal electronic structure, the point charge approximation fails. Although the diffuse charge model is more realistic as compared with the point charges, there still exists some arbitrariness in the choice of the charge size for every particular atom, and some parameterization work might be needed in order to choose proper effective charge sizes for different atom types. An important factor which has been left out in our calculations is the electronic polarizability of the protein environment within the retinal binding site. Some attempts have already been made to incorporate electronic polarizabilities of indivdual protein groups in the semi-empirical calculation of the electronic excitations of the bacteriochlorophyll b dimer in the photosynthetic reaction center of Rhodopseudomanas viridis [92]. These types of calculations need to be extended now to ab initio treatments of an arbitrary chromophore in situ.

It should be emphasized that proper descriptions of the physical properties and chemical transformation of in situ chromophores requires an accurate representation of the fluctuating thermal bath. An essential task is the description of the protein environment not in terms of one particular structure, but rather as an ensemble of different structures which are close in free energy but may possess quite different features resulting in a different photoexcitation response. A first step in this direction has already been taken in the work presented here as well as in modelling of multiple initial photoisomerization events for an ensemble of structures taken from a single dynamical trajectory [95]. However, more extended ensembles may be 
needed, which incorporate structures that are not linked by a single molecular dynamics trajectory on a picosecond time scale. 


\section{Acknowledgements}

The research was carried out at the Resource for Concurrent Biological Computing at the University of Illinois, funded by the National Institutes of Health (grant P41RR05969) and

by the Roy J. Carver Charitable Trust. The majority of simulations were done using Silicon Graphics and Hewlett-Packard workstations operated by the Resource. 


\section{References}

[1] H. G. Khorana. J. Biol. Chem., 263:7439-7442, 1988.

[2] R. R. Birge. Ann. Rev. Phys. Chem., 41:683-733, 1990.

[3] R. R. Birge. Biochim. Biophys. Acta, 1016:293-327, 1990.

[4] R. A. Mathies, S. W. Lin, J. B. Ames, and W. T. Pollard. Ann. Rev. Biochem. Bioeng., 20:491-518, 1991.

[5] J. K. Lanyi. J. Bioenerg. Biomemb., 24:169-179, 1992.

[6] D. Oesterhelt, J. Tittor, and E. Bamberg. J. Bioenerg. Biomemb., 24:181-191, 1992.

[7] T. Ebrey. Light energy transduction in bacteriorhodopsin. In M. Jacobson, editor, Thermodynamics of Membranes, Receptors and Channels, pages 353-387. CRC Press, New York, 1993.

[8] Klaus Schulten and Paul Tavan. Nature, 272:85-86, 1978.

[9] T. Mogi, L. J. Stern, B. H. Chao, and H. G. Khorana. J. Biol. Chem., 264:14192-14196, 1989.

[10] T. Mogi, T. Marti, and H. G. Khorana. J. Biol. Chem., 264:14197-14201, 1989.

[11] M. S. Braiman, T. Mogi, T. Marti, L. J. Stern, H. G. Khorana, and K. J. Rothschild. Biochemistry, 27:8516-8520, 1988.

[12] L. J. Stern and H. G. Khorana. J. Biol. Chem., 264:14202-14208, 1989. 
[13] K. Gerwert, B. Hess, J. Soppa, and D. Oesterhelt. Proc. Natl. Acad. Sci. USA, 86:49434947, 1989.

[14] K. Ohno, Y. Takeuchi, and M. Yoshida. Biochim. Biophys. Acta, 462:575-582, 1977.

[15] G. Orlandi and Klaus Schulten. Chem. Phys. Lett., 64:370-374, 1979.

[16] G. Harbison, O. Smith, J. Pardoen, C. Winkel, J. Lugtenburg, J. Herzfeld, R. Mathies, and R. Griffin. Proc. Natl. Acad. Sci. USA, 81:1706-1709, 1984.

[17] S. Smith, A. Myers, J. Pardoen, C. Winkel, P. Mulder, J. Lugtenburg, and R. Mathies. Biophysics, 81:2055-2059, 1984.

[18] N. Livnah and M. Sheves. J. Am. Chem. Soc., 115:351, 1993.

[19] O. Kalisky, C. R. Goldschmidt, and M. Ottolenghi. Biophys. J., 19:185-189, 1977.

[20] W. Sperling, C. Rafferty, K. Kohl, and N. Dencher. FEBS Lett., 97:129-132, 1979.

[21] T. Iwasa, F. Tokunaga, and T. Yoshizawa. Photochem. Photobiol., 33:539-545, 1981.

[22] S. P. Balashov, F. F. Litvin, and V. A. Sineshchekov. Sov. Sci. Rev. D. Physiochem. Biol., 8:1-61, 1988.

[23] K. Bryl, M. Taiji, M. Yoshizava, and T. Kobayashi. Photochem. Photobiol., 56:10131018, 1992.

[24] C. Gergely, C. Ganea, and G. Varo. Biophys. J., 67:855-861, 1994.

[25] R. Henderson and P. N. T. Unwin. Nature, 257:28-32, 1975. 
[26] R. Henderson, J. M. Baldwin, T. A. Ceska, F. Zemlin, E. Beckmann, and K. H. Downing. J. Mol. Biol., 213:899-929, 1990.

[27] Y. A. Ovchinnikov, N. G. Abdulaev, M. Y. Feigina, A. V. Kiselev, and N. A. Lobanov. FEBS Lett., 100:219-224, 1979.

[28] H. G. Khorana, G. E. Gerber, W. C. Herlihy, C. P. Gray, and R. J. Anderegg. Proc. Natl. Acad. Sci. USA, 76:5046-5050, 1979.

[29] Marco Nonella, Andreas Windemuth, and Klaus Schulten. J. Photochem. Photobiol., 54(6):937-948, 1991.

[30] Feng Zhou, Andreas Windemuth, and Klaus Schulten. Biochemistry, 32(9):2291-2306, 1993.

[31] William Humphrey, Ilya Logunov, Klaus Schulten, and Mordechai Sheves. Biochemistry, 33:3668-3678, 1994.

[32] Ilya Logunov, William Humphrey, Klaus Schulten, and Mordechai Sheves. Biophys. J., 68:1270-1282, 1995.

[33] M. Ferrand, G. Zaccai, M. Nina, J.C. Smith, C. Etchesbest, and B. Roux. FEBS Lett., 327:256-260, 1993.

[34] M. Nina, B. Roux, and J. Smith. BJ, 68:25-39, 1995.

[35] C. Scharnagl, J. Hettenkofer, and S. Fisher. JPC, 99:7787-7800, 1995.

[36] A. Warshel. Nature, 260:679-683, 1976. 
[37] A. Warshel. Proc. Natl. Acad. Sci. USA, 75:2558-2562, 1978.

[38] A. Warshel, Z. T. Chu, and J.-K. Hwang. Chem. Phys., 158:303-314, 1991.

[39] K. Nakanishi, M. Arnaboldi, V. Baloghnair, and B. Honig. J. Am. Chem. Soc., 102:7945-7947, 1980.

[40] Klaus Schulten, Uri Dinur, and Barry Honig. J. Chem. Phys., 73(8):3927-3935, 1980.

[41] Paul Tavan, Klaus Schulten, and Dieter Oesterhelt. Biophys. J., 47:415-430, 1985.

[42] Paul Tavan, Klaus Schulten, Wolfgang Gärtner, and Dieter Oesterhelt. Biophys. J., 47:349-356, 1985.

[43] H. Gilson and B. Honig. JACS, 110:1943-1350, 1988.

[44] Y. Beppu and T. Kakitani. Photochem. Photobiol., 59:660-669, 1994.

[45] Bruce S. Hudson, Bryan E. Kohler, and Klaus Schulten. Linear polyene electronic structure and potential surfaces. In Edward C. Lim, editor, Excited States, volume 6, pages 1-95. Academic Press, 1982.

[46] Paul Tavan and Klaus Schulten. Phys. Rev. B, 36(8):4337-4358, 1987.

[47] P. Du and R. Davidson. J. Phys. Chem., 94:7013-1020, 1990.

[48] R. Graham and K. Freed. J. Chem. Phys., 96:1304-1316, 1992.

[49] S. Rai and R. Buenker. Indian Jornal of Chemistry, 31:215-218, 1992. 
[50] L. Serrano-Andreas, M. Merchan, I. Nebot-Gil, I. Nebot-Gil, and B. Roos. J. Chem. Phys., 98:3151-3162, 1993.

[51] M. Olivucci, F. Bernardi, P. Celani, I. Ragazos, and M. A. Robb. J. Am. Chem. Soc., 116:1077-1085, 1994.

[52] Klaus Schulten and Martin Karplus. Chem. Phys. Lett., 14(3):305-309, 1972.

[53] Paul Tavan and Klaus Schulten. J. Chem. Phys., 70(12):5407-5413, 1979.

[54] M. Olivucci, F. Bernardi, S. Ottani, and M. A. Robb. J. Am. Chem. Soc., 116:2034$2048,1994$.

[55] V. Bonacic-Koutecky, K. Schoffel, and J. Michl. Theoret. Chim. Acta, 72:459-474, 1987.

[56] B. Roos, P. Taylor, and P. Siegbahn. Chem. Phys., 48:157, 1980.

[57] B. Lengsfield. J. Chem. Phys., 73:382, 1980.

[58] I. Ragos, M. Robb, F. Bernardi, and M. Ollivuci. Chem. Phys. Lett., 197:217, 1992.

[59] M. Ollivuci, I. Ragazos, F. Bernardi, and M. Robb. J. Am. Chem. Soc., 115:3710-3721, 1993.

[60] S. Langhoff and E. Davidson. Int. J. Quantum Chem., 8:61, 1974.

[61] P. Hay and I. Shavitt. J. Chem. Phys., 60:2865, 1974.

[62] J. Pople, R. Seeger, and R. Krishnan. Int. J. Quantum Chem. Symp., 11:149, 1977.

[63] Paul Tavan and Klaus Schulten. J. Chem. Phys., 72(6):3547-3576, 1980. 
[64] Paul Tavan and Klaus Schulten. J. Chem. Phys., 85(11):6602-6609, 1986.

[65] K. Raghavachari and J. Pople. Int. J. Quantum Chem., 20:167, 1981.

[66] L. Serrano-Andreas, M. Merchan, I. Nebot-Gil, B. Roos, and M. Fulscher. J. Am. Chem. Soc., 115:6184-6197, 1993.

[67] B. Roos, M. Merchan, R. McDiarmid, and X. Xing. J. Am. Chem. Soc., 116:5927-5936, 1993.

[68] M. Merchan, E. Orti, and B. Roos. Chem. Phys. Lett., 226:27-36, 1994.

[69] G. J. Turner, L. J. W. Miercke, T. E. Thorgeirsson, D. S. Kliger, M. C. Betlach, and R. M. Stroud. Biochemistry, 32:1332-1337, 1993.

[70] S. P. Balashov, R. Govindjee, M. Kono, E. Imasheva, E. Lukashev, T. G. Ebrey, R. K. Crouch, D. R. Menick, and Y. Feng. Biochemistry, 32:10331-10343, 1993.

[71] T. Kobayashi, M. Terauchi, T. Kouyama, M. Yoshizawa, and M. Taiji. SPIE J., 1403:407-416, 1990.

[72] L. Song, M. A. El-Sayed, and J. K. Lanyi. Science, 261:891-894, 1993.

[73] J. Chandrasekhar, S. F. Smith, and W. L. Jorgensen. J. Am. Chem. Soc., 107:154, 1985.

[74] J. D. Madura and W. L. Jorgensen. J. Am. Chem. Soc., 108:2517, 1986.

[75] S. Miertus, E. Scrocco, and J. Tomasi. Chem. Phys., 55:117, 1981. 
[76] S. Miertus and J. Tomasi. Chem. Phys., 65:239, 1982.

[77] D. Tannor, B. Marten, R. Murphy, R. Friesner, D. Sitkoff, A. Nicholls, M. Rignalda, W. Doddard, and B. Honig. J. Am. Chem. Soc., 116:11875-11882, 1994.

[78] E. Clementi. Computational aspects for large chemical systems. In Lecture Notes in Chemistry. Springer, New York, 1980.

[79] B. T. Thole and P. T. van Duijnen. Phys. Chem., 71:211, 1982.

[80] U. C. Singh and P. A. Kollman. J. Comp. Chem., 7:718, 1986.

[81] M. J. Field, P. A. Bash, and M. Karplus. J. Comp. Chem., 11(6):700-733, 1990.

[82] P. A. Bash, M. J. Field, R. C. Davenport, G. A. Petsko, D. Ringe, and M. Karplus. Biochemistry, 30:5826-5832, 1991.

[83] J. Gao and X. Xia. Science, 258:631-635, 1992.

[84] M. Nina, J.C. Smith, and R. Roux. J. Mol. Struc., 1993.

[85] P. Du, S. C. Racine, and R. Davidson. J. Phys. Chem., 94:3944-3951, 1990.

[86] A.A. Kornyshev and S. Leikin. Phys. Rev. A, 40:6431-6437, 1989.

[87] Arieh Warshel. Computer Modelling of Chemical Reactions in Enzymes and Solutions. John Wiley and Songs, Inc., New York, 1991.

[88] A. Warshel and J. Aqvist. Ann. Rev. Biophys. Biophys. Chem., 20:267, 1991.

[89] A. Warshel. Curr. Opinion Struct. Biol., 2:230-236, 1992. 
[90] J. Aquist and A. Warshel. Chem. Rev., 93:2523-2544, 1993.

[91] H. Berendsen and J. Mavri. JPC, 97:13464-13468, 1993.

[92] M. Thompson and G. Schenter. JPC, 99:6374-6386, 1995.

[93] S. Sheiner. Advances in Biophysical Chemistry, 3:119-159, 1993.

[94] J. Lanyi. Nature, 375:461-463, 1995.

[95] William Humphrey, Dong Xu, Klaus Schulten, and Mordechai Sheves. J. Phys. Chem. Submitted. [Beckman Institute Technical Report TB-95-05]. 


\section{Table and Figure Captions}

Figure 3: Photocycles of bacteriorhodopsin. Shown are the $\mathrm{bR}_{568}$ and $\mathrm{bR}_{548}$ photocycles. Superscripts on intermediates indicate measured absorption maxima of these states. Isomerization configurations of retinal in every state are indicated in brackets. Steps involving photo- or thermally- induced isomerization of retinal, as well as proton transfer reactions are marked properly.

Figure 2: (a) Ribbon diagram of bacteriorhodopsin, showing residues which are implicated in the proton pump mechanism. Water molecules placed within the protein interior are represented as solid spheres. Helices C and D are shown as thin ribbons to reveal the retinal binding site. (1b) Time dependence of the retinal dihedral energy. (2b) Time dependence of the electrostatic interaction energy between retinal and the rest of the protein.

Figure 1: (a) Numbering scheme of the retinal chromophore bound via a protonated Schiff base linkage to a lysine side chain. (b) Part of the retinal chromophore utilized in the in situ ab initio calculations.

Figure 5: (a) Spectra of bacteriorhodopsin: experimental spectrum of the native bR pigment, calculated spectrum of the native bR pigment, and calculated spectrum of the D85N mutant of bR. (b) Retinal binding sites of the native bR pigment and its D85N mutant.

Figure 4: (1a) Time dependence of the excitation energy of in situ retinal. (1b) Autocorrelation function for the excitation energy of in situ retinal. (2a) Time dependence 
of the distance between the Schiff base nitrogen and the oxygen of a hydrogen bond forming water molecule. (2b) Autocorrelation function for the distance between the Schiff base nitrogen and the oxygen of a hydrogen bond forming water molecule.

Figure 6: Sequence of retinal conformations which correspond to the dark adaptation process in bacteriorhodopsin. Shown are two possible pathways for the dark adaptation. It is currently believed that dark adaptation occurs via protonation of the Asp-85 side group (the bottom pathway).

Figure 7: (a) Retinal ground state potential surfaces corresponding to the dark adaptation process. Shown are in situ and in vacuo potential surfaces obtained from the same sequence of retinal conformations. (b) Effect of the Coulomb interactions in the bR binding site on the in situ dark adaptation potential surface. The 'classical' curve (top) accounts for the pair-wise electrostatic interactions between retinal and the rest of the protein in the course of isomerization. The 'quantum' curve (bottom) corresponds to the difference of the computed ab initio in situ and in vacuo potential curves.

Figure 8: Computed distribution of the in situ barriers corresponding to the dark adaptation process in case of two different protonation states of Asp-85. 
(a)

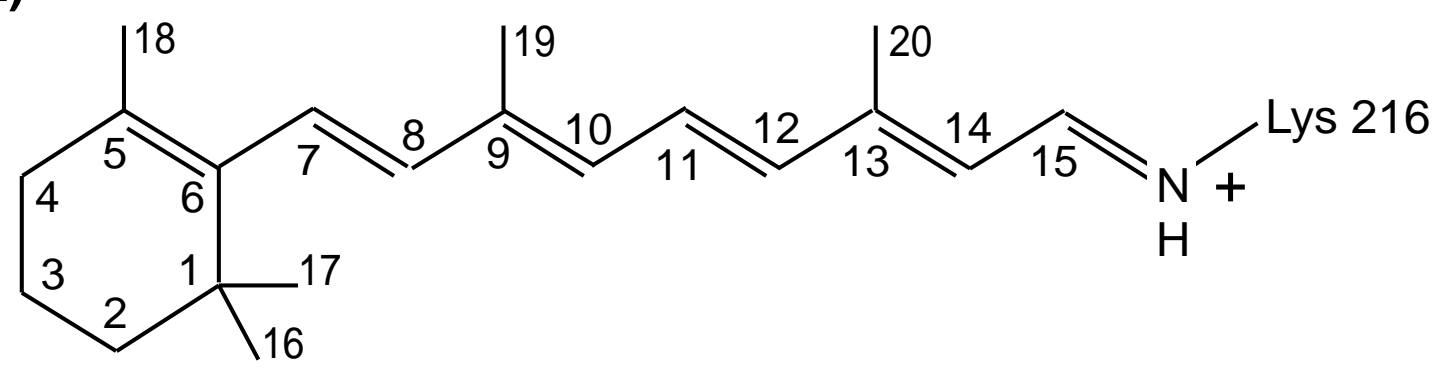

(b)

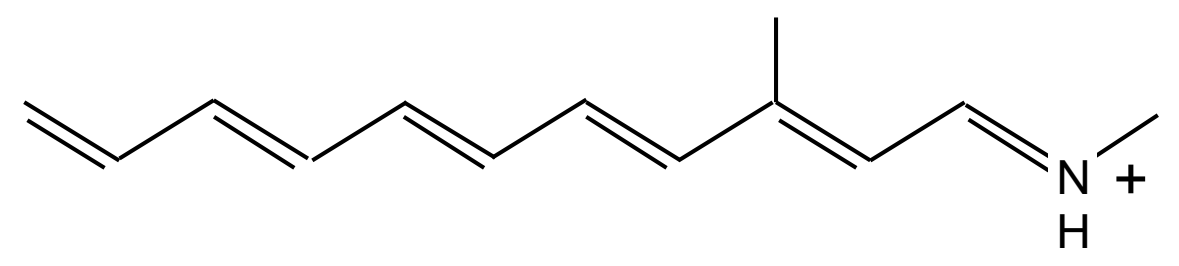

Figure 1:

(a) Numbering scheme of the retinal chromophore bound via a protonated Schiff base linkage to a lysine side chain. (b) Part of the retinal chromophore utilized in the in situ ab initio calculations. 
(a)

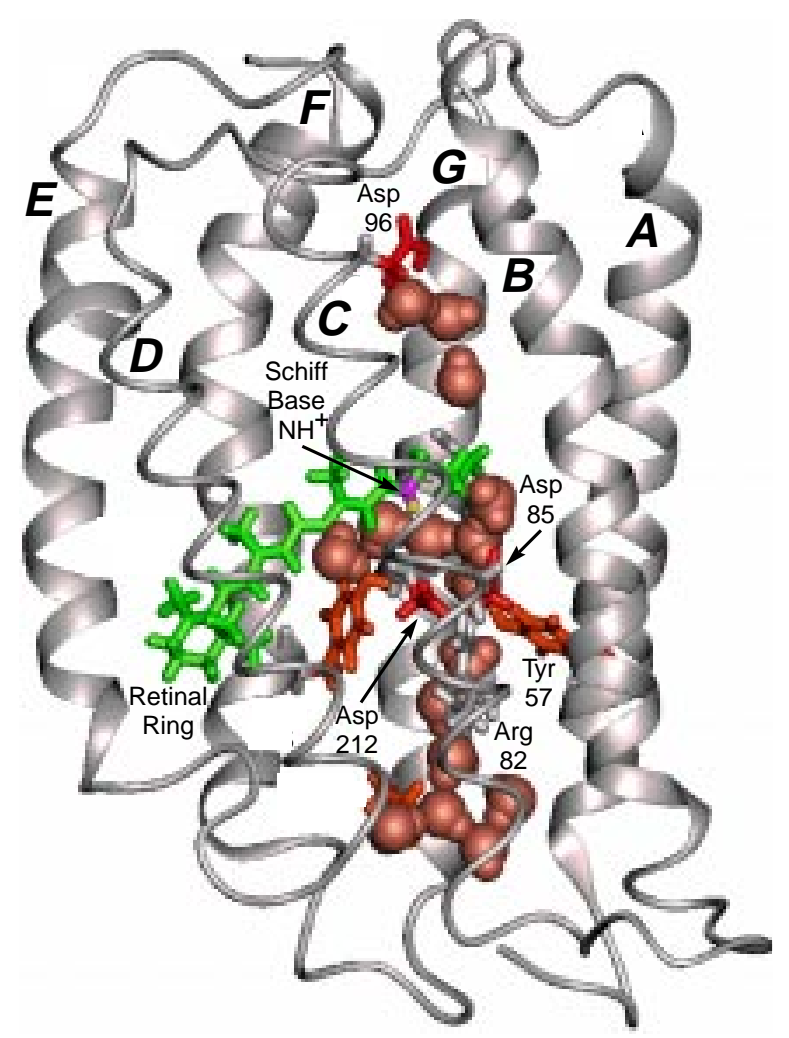

(1b)

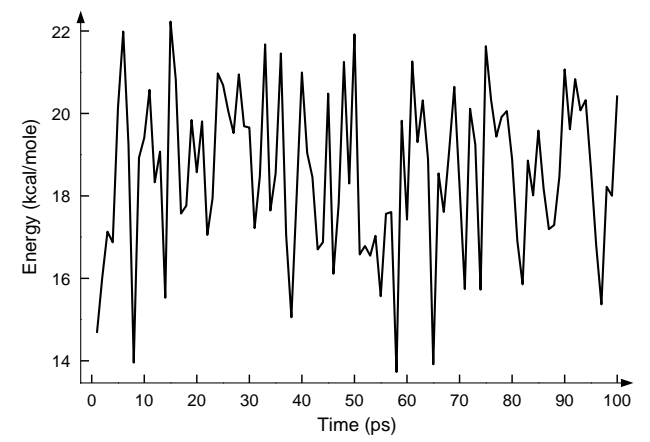

(2b)

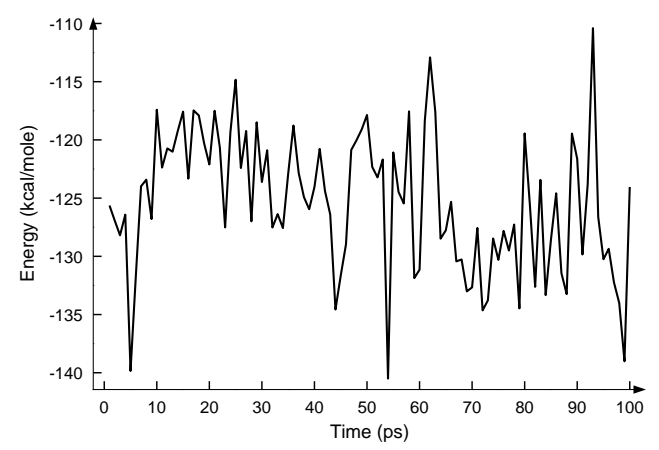

Figure 2:

(a) Ribbon diagram of bacteriorhodopsin, showing residues which are implicated in the proton pump mechanism. Water molecules placed within the protein interior are represented as solid spheres. Helices $\mathrm{C}$ and $\mathrm{D}$ are shown as thin ribbons to reveal the retinal binding site. (1b) Time dependence of the retinal dihedral energy. (2b) Time dependence of the electrostatic interaction energy between retinal and the rest of the protein. 


\section{trans photocycle}

\section{cis photocycle}

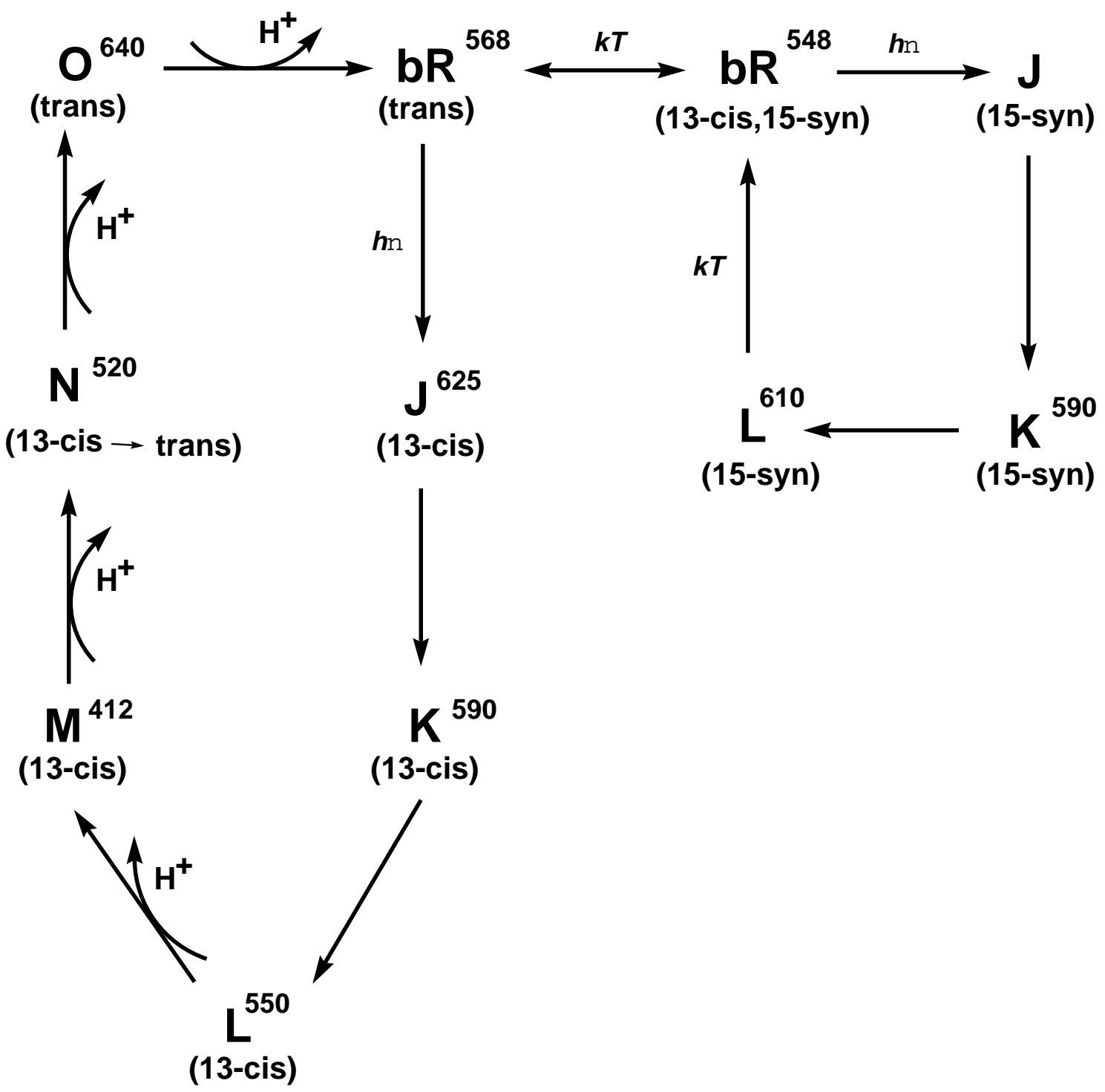

Figure 3:

Photocycles of bacteriorhodopsin. Shown are the $b_{568}$ and $b R_{548}$ photocycles. Superscripts on intermediates indicate measured absorption maxima of these states. Isomerization configurations of retinal in every state are indicated in brackets. Steps involving photo- or thermally- induced isomerization of retinal, as well as proton transfer reactions are marked properly. 
(1a)

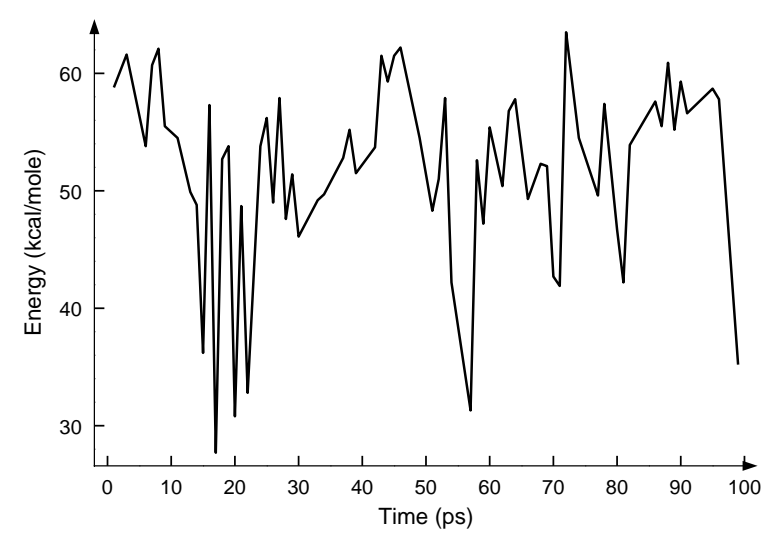

(1b)

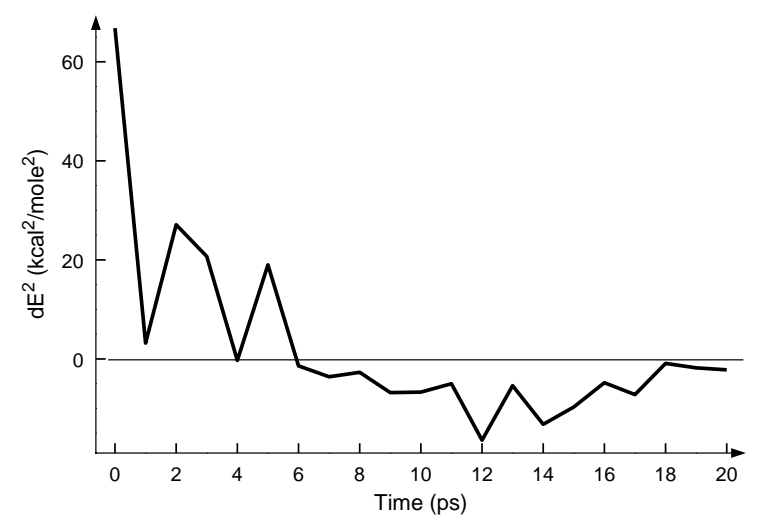

(2a)

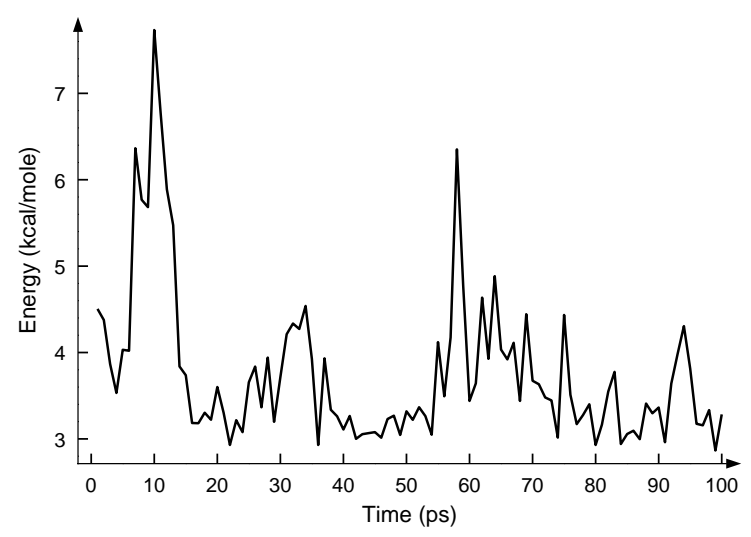

(2b)

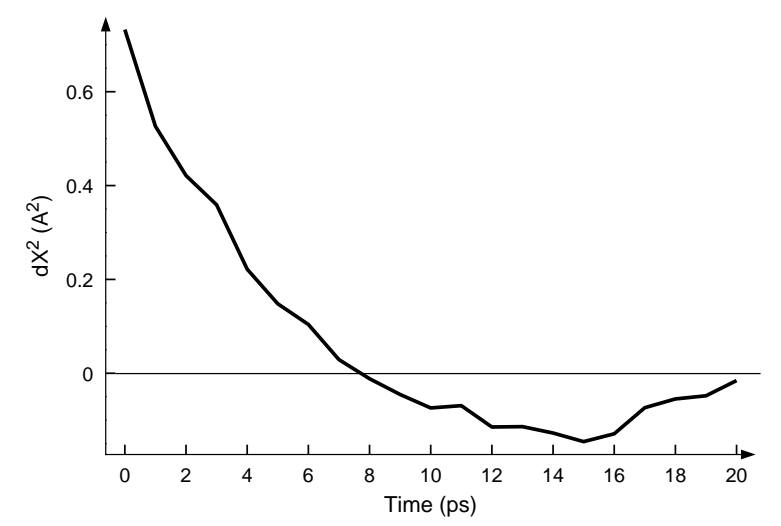

Figure 4:

(1a) Time dependence of the excitation energy of in situ retinal. (1b) Autocorrelation function for the excitation energy of in situ retinal. (2a) Time dependence of the distance between the Schiff base nitrogen and the oxygen of a hydrogen bond forming water molecule. (2b) Autocorrelation function for the distance between the Schiff base nitrogen and the oxygen of a hydrogen bond forming water molecule. 
(a)

\section{Absorption Spectra of Bacteriorhodopsin}

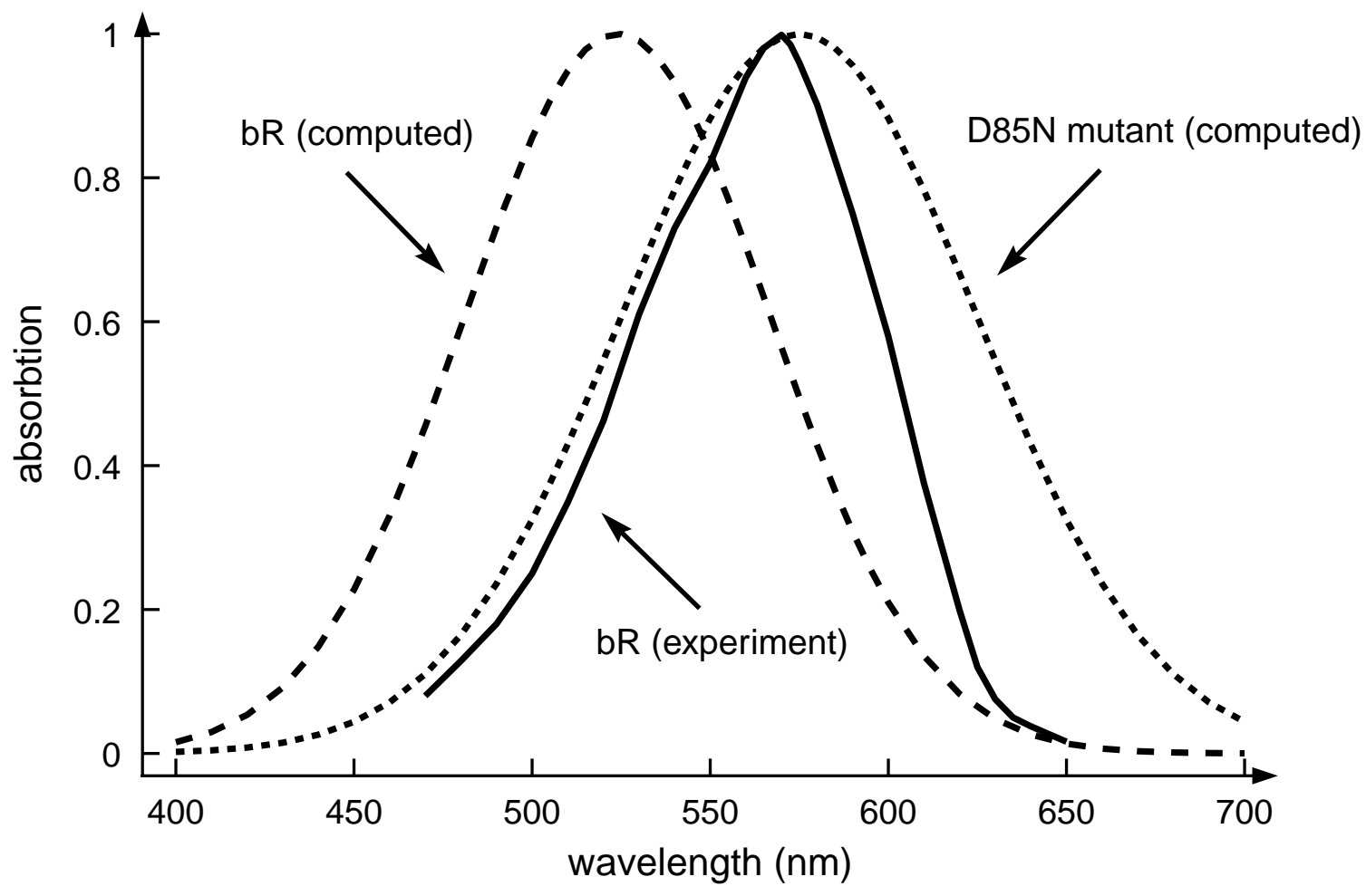

(b)

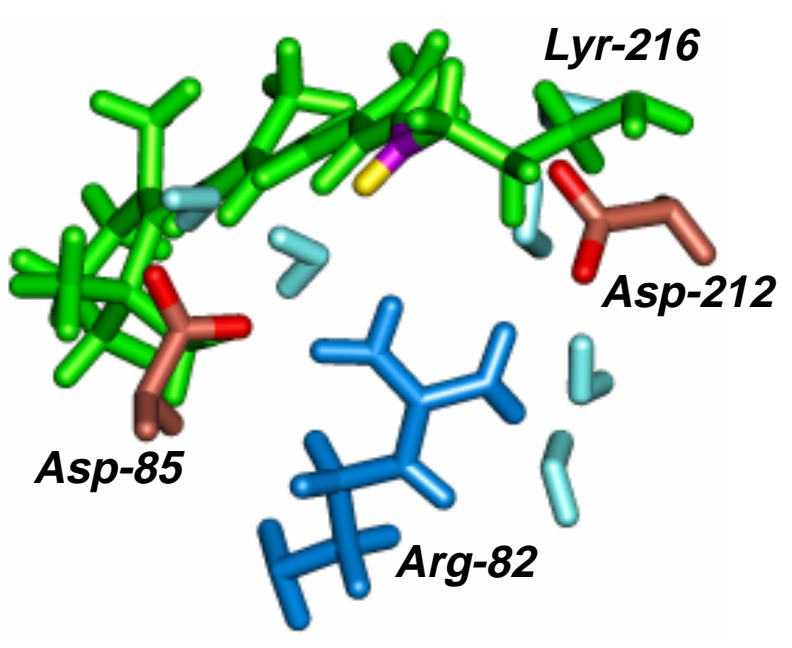

D85N active site

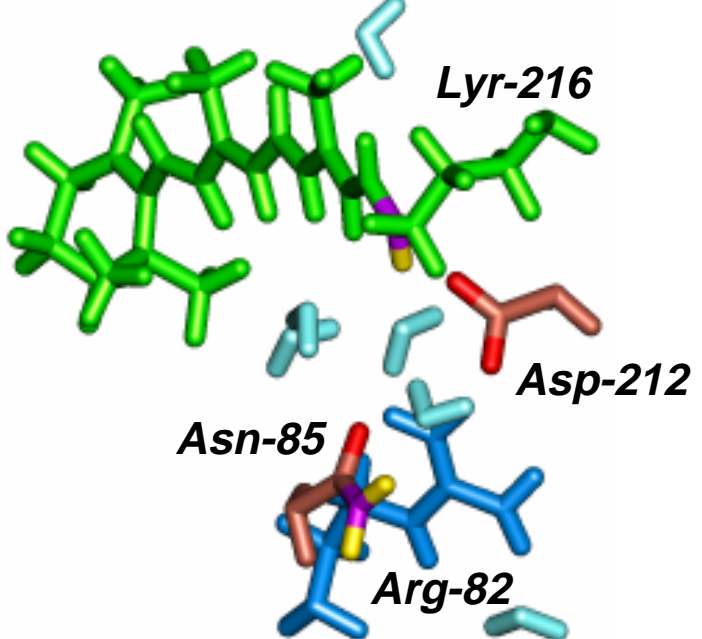

Figure 5:

(a) Spectra of bacteriorhodopsin: experimental spectrum of the native bR pigment, calculated spectrum of the native bR pigment, and calculated spectrum of the D85N mutant of bR. (b) Retinal binding sites of the native bR pigment and its D85N mutant. 


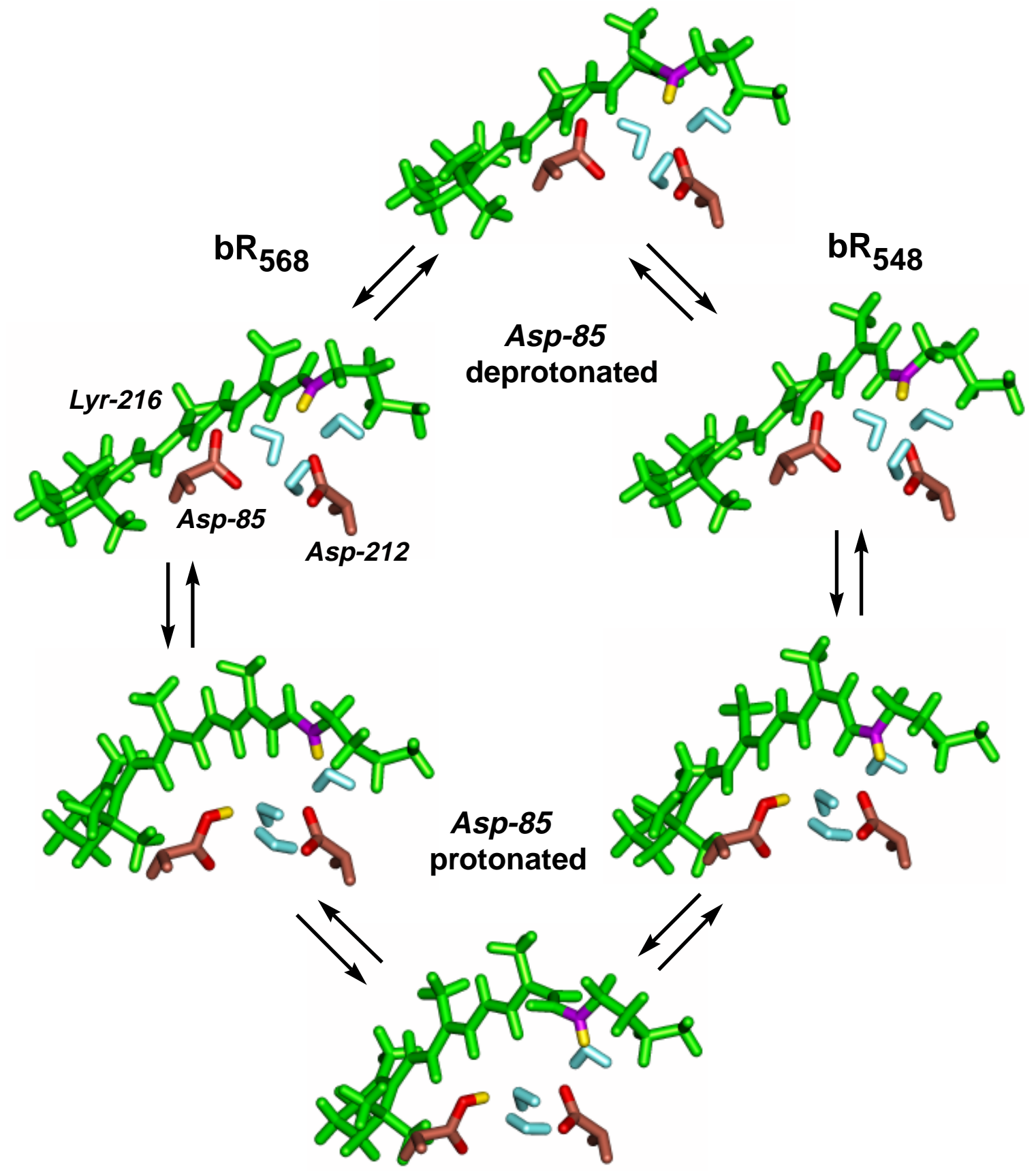

Figure 6:

Sequence of retinal conformations which correspond to the dark adaptation process in bacteriorhodopsin. Shown are two possible pathways for the dark adaptation. It is currently believed that dark adaptation occurs via protonation of the Asp-85 side group (the bottom pathway). 

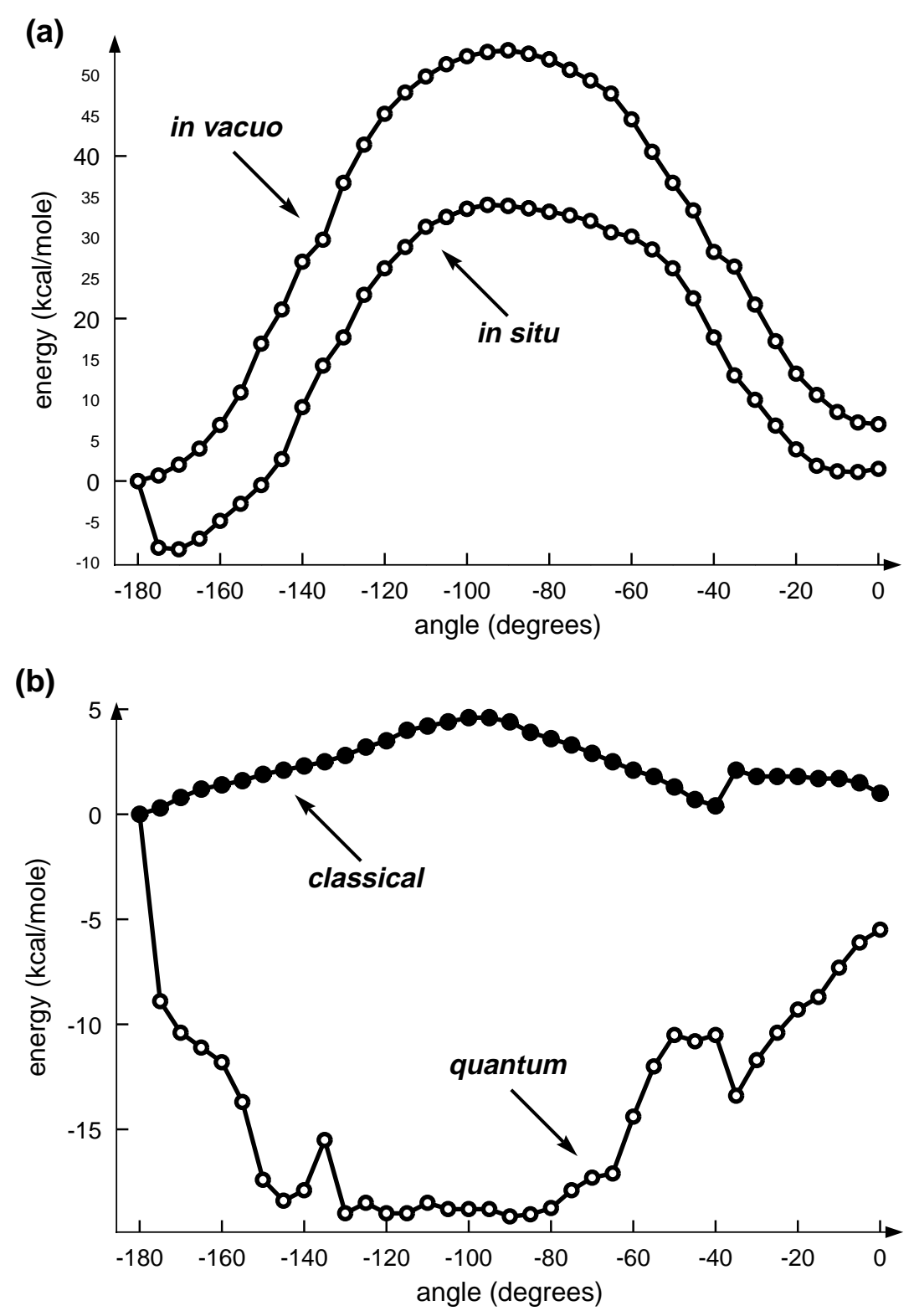

Figure 7:

(a) Retinal ground state potential surfaces corresponding to the dark adaptation process. Shown are in situ and in vacuo potential surfaces obtained from the same sequence of retinal conformations. (b) Effect of the Coulomb interactions in the bR binding site on the in situ dark adaptation potential surface. The 'classical' curve (top) accounts for the pairwise electrostatic interactions between retinal and the rest of the protein in the course of isomerization. The 'quantum' curve (bottom) corresponds to the difference of the computed $a b$ initio in situ and in vacuo potential curves. 


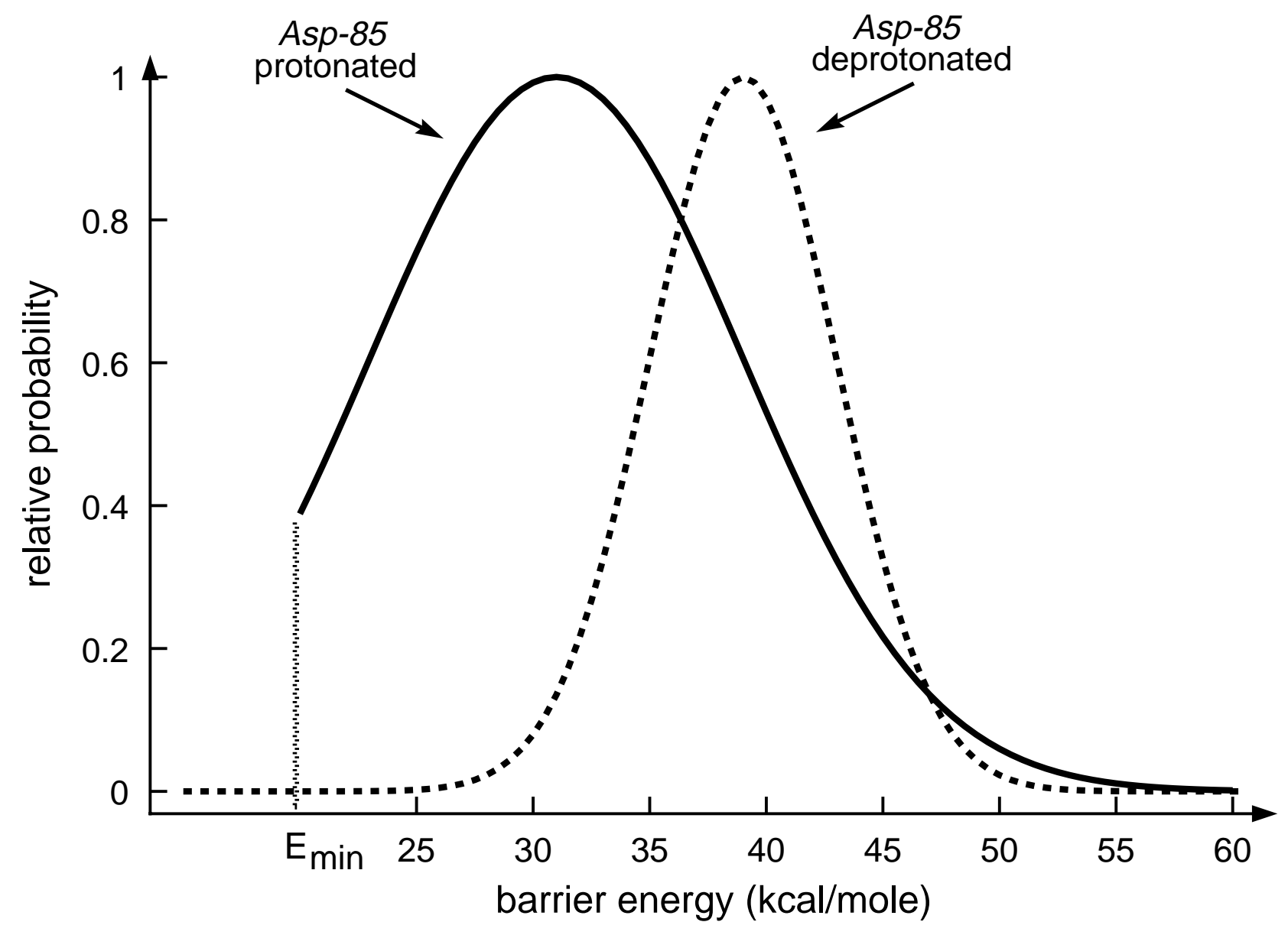

Figure 8:

Computed distribution of the in situ barriers corresponding to the dark adaptation process in case of two different protonation states of Asp-85. 